Research Paper

\title{
Difierential Genetic Expression in Large Versus Small Clear Cell Renal Cell Carcinoma: Results from Microarray Analysis
}

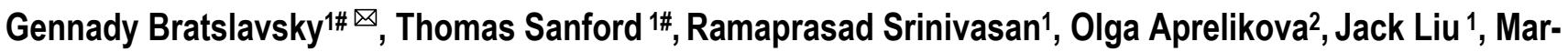 \\ tha Quezado ${ }^{3}$, Maria Merino ${ }^{3}$, W. Marston Linehan ${ }^{1}$
}

1. Urologic Oncology Branch, National Cancer Institute, Bethesda, MD, USA

2. Cell and Cancer Biology Branch, National Cancer Institute, National Institutes of Health, Bethesda, MD, USA

3. Surgical Pathology Branch, National Cancer Institute, National Institutes of Health, Bethesda MD, USA

\# Equal contribution

Corresponding author: Gennady Bratslavsky, M.D., Urologic Oncology Branch, National Cancer Institute, National Institutes of Health, Building 10, Room 1-5940, Bethesda, Maryland 20892-1414 USA. Phone: (301)496-6353; Fax: (301) 480-5626; E-mail: bratslag@mail.nih.gov or bratslag@upstate.edu

(C) Ivyspring International Publisher. This is an open-access article distributed under the terms of the Creative Commons License (http://creativecommons.org/ licenses/by-nc-nd/3.0/). Reproduction is permitted for personal, noncommercial use, provided that the article is in whole, unmodified, and properly cited.

Received: 2011.04.21; Accepted: 2011.05.12; Published: 2011.05.12

\begin{abstract}
Purpose: Tumor growth and progression requires multiple steps and genetic alterations. The molecular events that occur as tumors increase in size are unknown. Patients with von Hippel-Lindau (VHL) provide a unique opportunity to study molecular alterations during tumor growth as these patients develop multiple bilateral renal tumors. To better characterize biologic events associated with tumor growth, we evaluated the alterations in gene expression in large versus small renal tumors removed from the same kidney of the same individuals.

Experimental Design: We reviewed pathology reports from patients who underwent partial nephrectomies at the National Cancer Institute for multiple tumors. We identified II patients who fulfilled the following inclusion criteria: I) The patient must have had a surgical resection of more than one solid tumor from the same kidney during the same operation; 2) Among the solid tumors at least one must have been greater than $3 \mathrm{~cm}$ in the largest dimension and at least one less than $2 \mathrm{~cm} ; 3$ ) the nuclear Furhman grade for both larger and smaller solid tumors was identical; 4) a portion of each tumor was procured and snap frozen after surgical removal; 5) Hematoxylin and eosin staining of the frozen sample confirmed clear cell carcinoma to be present in at least $80 \%$ of the section.

Affymetrix platform and protocol for gene expression arrays were used. RNA from the frozen large and small tumor samples was extracted using Trizol-Chlorophorm method. The RNA was then reverse transcribed, labeled, fragmented, and hybridized on to an Affymetrix UI33 Plus 2.0 array that contains 54,000 probe sets representing 24,568 genes. Analysis included unsupervised clustering and chromosomal analysis. The paired t-test was performed to compare gene expression levels in small and large tumors. $\mathrm{P}<0.0 \mathrm{I}$ was considered statistically significant.

Results: Gene expression profiles were assessed for 22 tumors (I I patients). Upon unsupervised clustering the pairs with larger tumor volume difference clustered separately from pairs with smaller volume difference. Chromosomal analysis revealed few consistent changes other than reduced expression of chromosome 3p25 among all tumors. Paired t-test showed 860 differentially expressed genes in the TIb vs Tla group, a number far greater than ex-
\end{abstract}


pected due to chance alone. When analyzed by gene function, most differences were observed in genes involved in DNA replication and in cytokine signaling.

Conclusions: This study demonstrates that as tumors increase in size there is an increasing difference in gene expression. Unsupervised clustering analysis confirms that as the volume difference increases there are a distinct set of genes that are regulated either as a response to a tumor's growth or as an early event that causes the tumor to grow. While we did not observe chromosomal instability, we did note differences in expression of individual transcripts as tumors grew larger.

Key words: Renal cell carcinoma, tumor, size, microarray, kidney

\section{Introduction and Objectives}

During the last two decades, the molecular events that lead to the formation of renal cell carcinoma have been elucidated, particularly for the clear cell type (ccRCC). Most clear cell renal tumors form as the result of dysregulation of the von Hippel-Lindau tumor (VHL) suppressor gene with loss-of-function somatic mutations and epigenetic silencing that may be found in up to $90 \%$ of sporadic ccRCC (1). While the inciting genetic events that lead to the formation of ccRCC have been worked out in great detail, the events that give primary tumors the ability to grow and potentially gain aggressiveness remain poorly understood.

There is a strong relationship between the size of the primary tumor and likelyhood of metastasis. Frank et al demonstrated that estimated cancer specific survival rates for tumors $>7 \mathrm{~cm}$ were $15-25 \%$ higher than for tumors that were less than $<7 \mathrm{~cm}$ following nephrectomy (2). In a separate study from the $\mathrm{NCI}$ of a cohort of patients with inherited germline mutations of the $V H L$ gene, there have been no cases of metastasis of tumors less than $3 \mathrm{~cm}$ (3). To explore the genetic events involved in the development and progression of ccRCC as tumors grow larger, we surveyed the global gene expression profiles of tumors of different sizes in patients with confirmed germline VHL gene mutations. By using tumors of different sizes removed from the same patient, we aimed to study the differences in genetic profiles associated with increase in tumor size.

\section{Materials/Methods}

\section{Patient selection}

Pathology reports from patients who underwent partial nephrectomies for multiple renal tumors at the National Cancer Institute were reviewed. Eleven patients fulfilled the following inclusion criteria: 1) The patient must have had a surgical resection of more than one solid tumor from the same kidney during the same operation; 2) Among the solid tumors at least one must have been greater than $3 \mathrm{~cm}$ in the largest dimension while the other removed solid tumor was less than $2 \mathrm{~cm}$; 3) the nuclear Furhman grade for both larger and smaller solid tumors was identical; 4) a portion of each tumor was procured and snap frozen after surgical removal; 5) Hematoxylin and eosin staining of the frozen sample confirmed clear cell carcinoma to be present in at least $80 \%$ of the section. Original tumor volumes were calculated using the formula for an ellipsoid $\left(4 / 3 \Pi r^{3}\right)$. All patients had confirmed mutations in the VHL tumor suppressor gene. All patients were enrolled in an IRB approved NIH intramural research protocol.

A summary of the tumor sizes for the eleven patients included in this study can be seen in Table 1. Four pairs have at least 1 tumor $>4 \mathrm{~cm}$ (used to compare T1a vs T1b), and seven pairs have two tumors $<4 \mathrm{~cm}$ (used to compare T1a vs T1a).

An example of the multifocal renal tumors in a patient with VHL disease can be seen in the bivalved nephrectomy specimen in Figure 1 demonstrating multiple tumors of different sizes throughout the kidney.

\section{RNA Isolation}

Fifteen $20 \mu \mathrm{m}$ thick frozen sections from each tissue sample were homogenized in TRIZOL reagent (Invitrogen Life Techonolgies, Carlsbad, CA USA). Total RNA was extracted using a standard chloroform protocol followed by purification with the Qiagen RNeasy Mini Kit (QIAGEN Inc, Valencia, CA USA). RNA integrity was evaluated by using RNA 6000 Nano LabChips on an Agilent 2100 Bioanalyzer (Agilent Technologies, Foster City, CA USA). RNA purity was assessed by the ratio of spectrophotometric absorbance at 260 and $280 \mathrm{~nm}$ (A260/280nm) using NanoDrop ND-1000 (NanoDrop Inc, Wilmington, DE USA). All chips were prepared according to the manufacturer's instructions. Total RNA degradation was evaluated by reviewing the electropherograms and the RNA integrity number (RIN): only samples with preserved $18 \mathrm{~S}$ and $28 \mathrm{~S}$ peaks and RIN values greater than 7 were selected for gene expression analysis. 
Table I. Tumor sizes for the patients

\begin{tabular}{|c|c|c|c|c|c|}
\hline & Size & Tumor size $(\mathrm{cm})$ & Size Difference $(\mathrm{cm})$ & Tumor volume $\left(\mathrm{cc}^{3}\right)$ & Volume Difference $\left(\mathrm{cc}^{3}\right)$ \\
\hline \multirow[t]{2}{*}{ Patient 1} & large & 6 & 4.7 & 84.8 & 84.0 \\
\hline & small & 1.3 & & 0.8 & \\
\hline \multirow[t]{2}{*}{ Patient 2} & large & 5.5 & 3.7 & 25.9 & 24.5 \\
\hline & small & 1.8 & & 1.4 & \\
\hline \multirow[t]{2}{*}{ Patient 3} & large & 5.2 & 4 & 34.3 & 31.9 \\
\hline & small & 1.2 & & 2.4 & \\
\hline \multirow[t]{2}{*}{ Patient 4} & large & 4.5 & 2.5 & 24.7 & 22.0 \\
\hline & small & 2 & & 2.7 & \\
\hline \multirow[t]{2}{*}{ Patient 5} & large & 3.5 & 1.7 & 11.9 & 11.4 \\
\hline & small & 1.8 & & 0.5 & \\
\hline \multirow[t]{2}{*}{ Patient 6} & large & 3.5 & 2 & 9.2 & 8.9 \\
\hline & small & 1.5 & & 0.3 & \\
\hline \multirow[t]{2}{*}{ Patient 7} & large & 3.4 & 2.1 & 15.9 & 15.3 \\
\hline & small & 1.3 & & 0.6 & \\
\hline \multirow[t]{2}{*}{ Patient 8} & large & 3.1 & 1.1 & 11.8 & 10.3 \\
\hline & small & 2 & & 1.5 & \\
\hline \multirow[t]{2}{*}{ Patient 9} & large & 3 & 1 & 4.7 & 2.3 \\
\hline & small & 2 & & 2.4 & \\
\hline \multirow[t]{2}{*}{ Patient 10} & large & 3 & 1.6 & 7.9 & 6.8 \\
\hline & small & 1.4 & & 1.1 & \\
\hline \multirow[t]{2}{*}{ Patient 11} & large & 3 & 1.8 & 14.1 & 13.8 \\
\hline & small & 1.2 & & 0.3 & \\
\hline
\end{tabular}

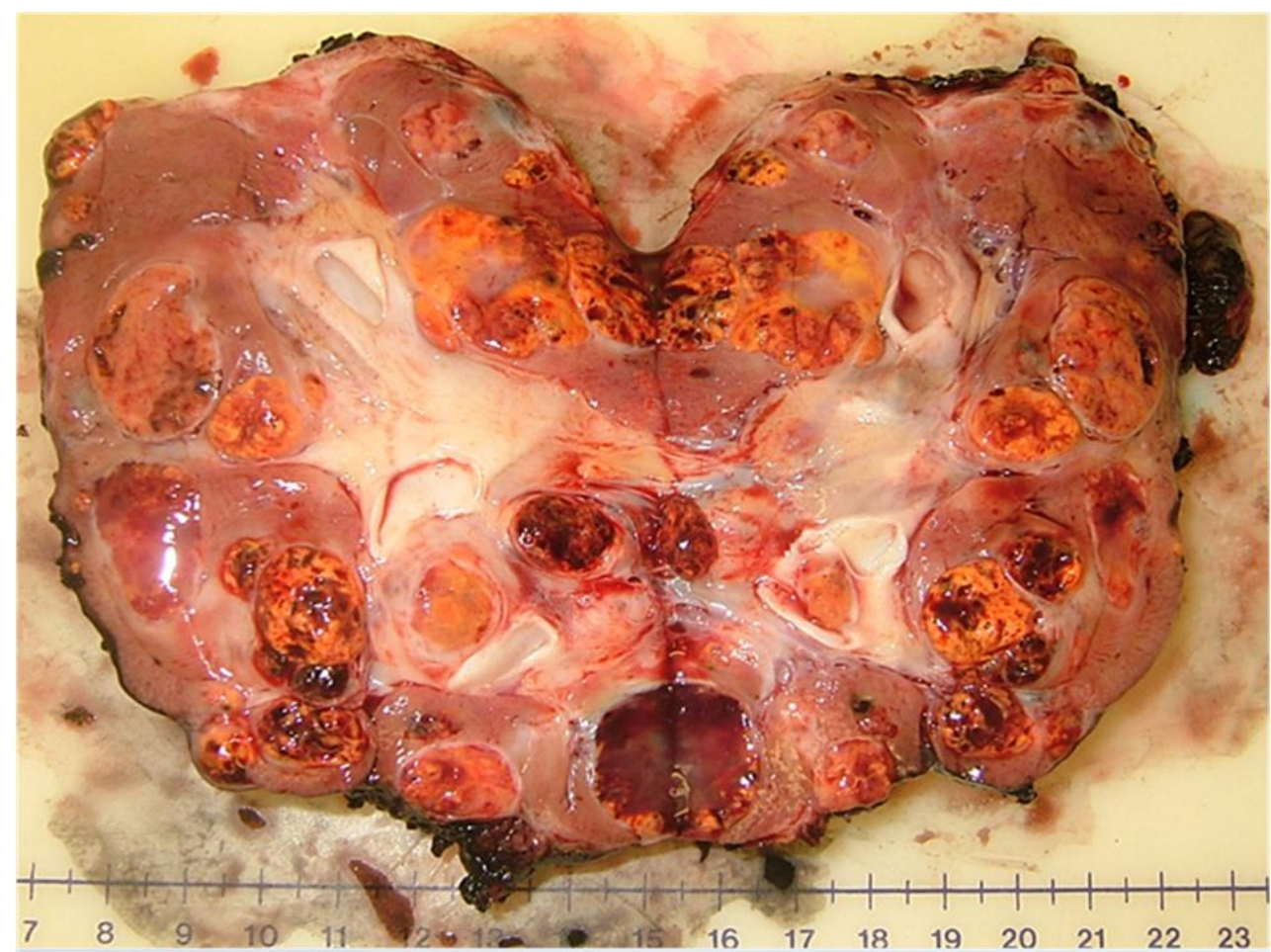

Figure I. Multiple renal tumors with a single renal unit: bivalved nephrectomy specimen from a patient with a germline mutation in the $\mathrm{VHL}$ gene. 


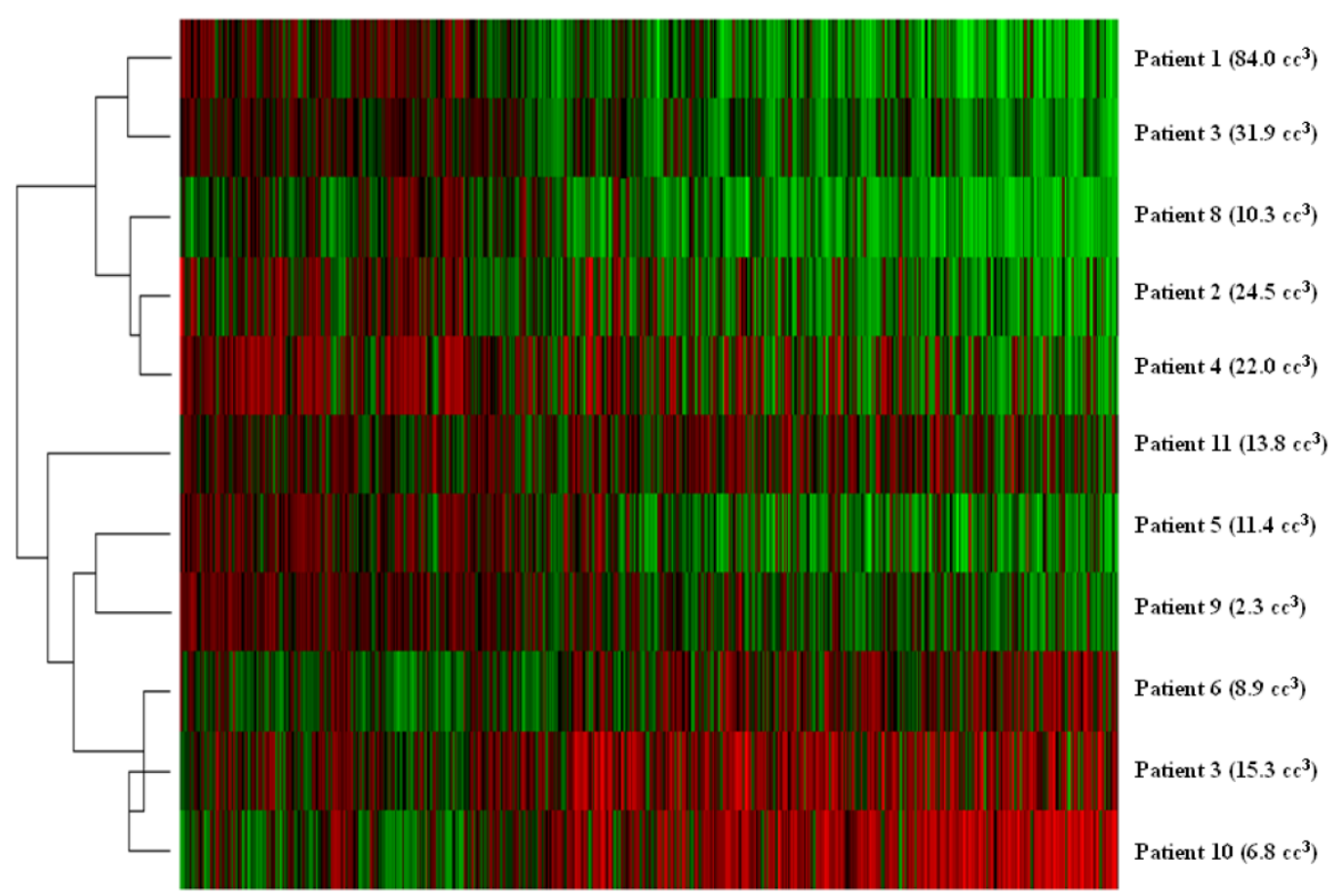

Figure 2. Hierarchical clustering of tumor pairs based on differences in size. The volume difference between larger and smaller tumor is listed in parenthesis for each patient.

\section{Microarray hybridization and Image Acquisition}

Messenger RNA expression levels were measured using the GeneChip ${ }^{\circledR}$ HG-U133 plus 2.0 platform following the manufacturer's protocol (Affymetrix Inc, Santa Clara, CA USA). Briefly, double-stranded cDNA was synthesized from $11 \mu \mathrm{g}$ of total RNA from each tumor sample using GeneChip Two-Cycle cDNA Target Synthesis Kit (Affymetrix Inc, Santa Clara, CA USA). After one round of amplifications, in vitro transcription was performed using IVT Labeling Kit (Affymetrix Inc, Santa Clara, CA USA) to synthesize biotin-labeled cRNA. An aliquot of 15ug labeled cRNA was fragmented and subsequently hybridized for 16 hours at $45 \mathrm{C}$ in a hybridization oven to a HG-U133 plus 2.0 olgionucleotide array. Arrays were washed and scanned with an Affymetrix GeneChip 3000 Scanner.

\section{Statistical Analysis}

Raw data (.CEL) files were imported into BRB array tools (4). The data were normalized using the RMA algorithm (5). Unsupervised clustering was performed using hierarchical clustering (1-correlation) based on the differences in size between large and small tumors. The data were then separated into two groups for supervised analysis: the first consisted of pairs of tumors that were both T1a $(<4 \mathrm{~cm})$, and the second group had one T1a tumor and one $\mathrm{T} 1 \mathrm{~b}$ (between 4 and $7 \mathrm{~cm}$ ) to evaluate the accepted size cut offs of AJCC staging. Differences in gene expression were analyzed using a paired t-test with random variance model set at 1000 permutations. Significant P-value was set at 0.01. BRB array tools was used to analyze which categories are over-represented relative to the prevalence of Gene Ontology categories on the array (4).

Data were then imported into the $\mathrm{R}$ environment for further analysis (www.r-project.org). Chromosomal abnormalities were assessed with a technique known as comparative genomic microarray analysis. This method has been shown to have good correlation with more traditional $\mathrm{CGH}$ techniques (6). For a common reference pool, a pool of normal renal parenchyma from the gene expression omnibus database (http://www.ncbi.nlm.nih.gov/geo/) was used (GSE7392).

\section{Results}

The results of unsupervised clustering based on the volume difference of the pairs can be seen in Figure 2. With one exception, the pairs with larger differences in volume clustered separately from pairs 
with smaller differences.

A schematic of the results of the paired t-test are observed in figure 3. The Affymetrix HGU133 plus 2.0 has 54,675 probe sets. The threshold for significance was set at 0.01 . Thus, we would expect approximately 550 false positives. In the paired analysis where both tumors were less than $4 \mathrm{~cm}$, there were 458 genes were differentially expressed, less than would be expected by chance. When tumors greater than $4 \mathrm{~cm}$ were compared to tumors less than $4 \mathrm{~cm}$ in the same patient, there were 890 differentially expressed genes identified, which is greater than the expected false positives. When we examined the gene ontology of the significant gene changes, we found that transcripts involved in cytokine production and DNA synthesis to be over-represented (Table 2).

The first part of the cGMA chromosomal analysis was an examination of the expression pattern for chromosome 3 . Since all the patients had a confirmed VHL mutation, as expected the level of expression was decreased in chromosome 3p25 in all samples when compared to normal renal parenchyma (Figure 4). Interestingly, there is was also a decrease in gene expression near chromosome 3p21. Full chromosomal analysis revealed that the expression profiles within each pair appeared similar. No consistent differences in expression on chromosomal level between large and small tumors were observed. (Figure 5).
Table 2. Gene ontology

\begin{tabular}{|c|c|c|}
\hline GO ID & GO Term & $\begin{array}{l}\text { Observed/ } \\
\text { Expected }\end{array}$ \\
\hline GO:0032602 & chemokine production & 8.08 \\
\hline GO:0042364 & $\begin{array}{l}\text { water-soluble vitamin biosynthetic } \\
\text { process }\end{array}$ & 6.79 \\
\hline GO:0051187 & cofactor catabolic process & 6.57 \\
\hline GO:0034381 & lipoprotein particle clearance & 6.47 \\
\hline GO:0009110 & vitamin biosynthetic process & 5.5 \\
\hline GO:0006306 & DNA methylation & 5.39 \\
\hline GO:0006305 & DNA alkylation & 5.39 \\
\hline GO:0007031 & peroxisome organization & 4.85 \\
\hline GO:0031047 & gene silencing by RNA & 4.53 \\
\hline GO:0006304 & DNA modification & 4.04 \\
\hline GO:0030336 & negative regulation of cell migration & 3.99 \\
\hline GO:0001935 & endothelial cell proliferation & 3.93 \\
\hline GO:0016458 & gene silencing & 3.85 \\
\hline GO:0043542 & endothelial cell migration & 3.83 \\
\hline GO:0009062 & fatty acid catabolic process & 3.83 \\
\hline GO:0045765 & regulation of angiogenesis & 3.7 \\
\hline GO:0051271 & negative regulation of cell motion & 3.64 \\
\hline GO:0043414 & biopolymer methylation & 3.45 \\
\hline GO:0045762 & $\begin{array}{l}\text { positive regulation of adenylate } \\
\text { cyclase activity }\end{array}$ & 3.38 \\
\hline GO:0019362 & pyridine nucleotide metabolic process & 3.31 \\
\hline GO:0022618 & ribonucleoprotein complex assembly & 3.17 \\
\hline GO:0006733 & $\begin{array}{l}\text { oxidoreduction coenzyme metabolic } \\
\text { process }\end{array}$ & 3.17 \\
\hline GO:0031281 & positive regulation of cyclase activity & 3.16 \\
\hline GO:0051349 & positive regulation of lyase activity & 3.1 \\
\hline
\end{tabular}

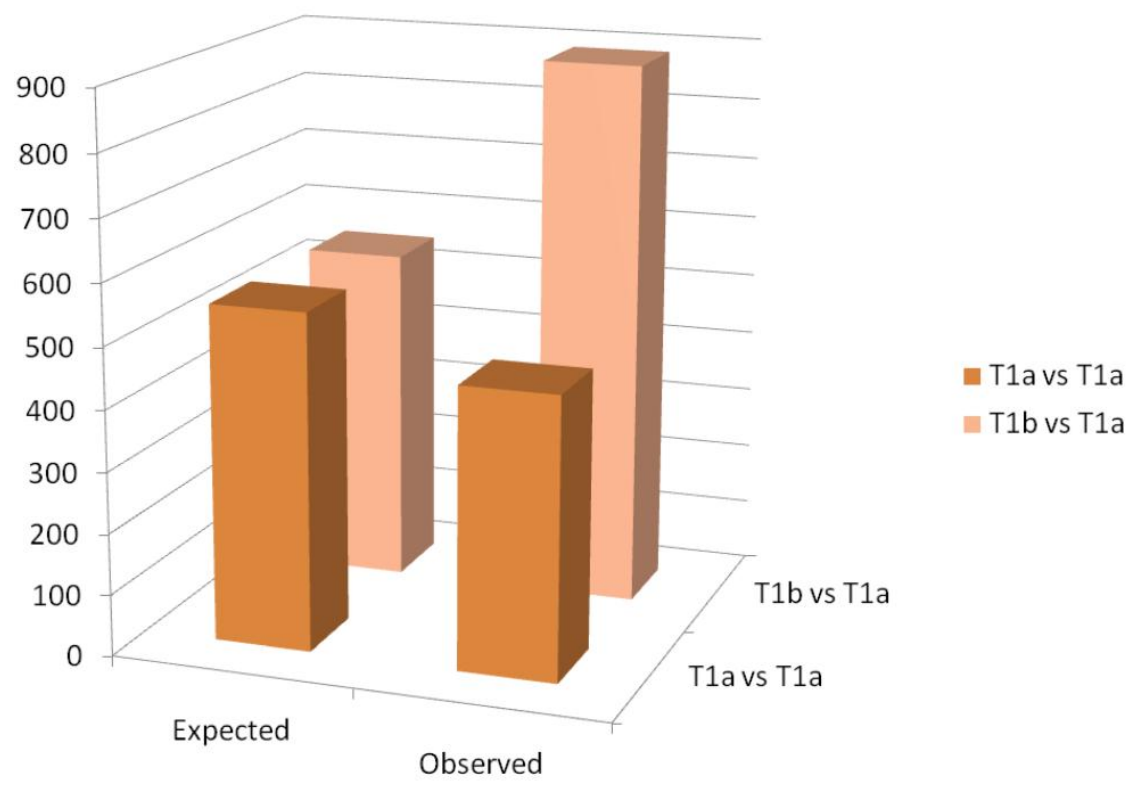

Figure 3. Column graph of the observed and expected gene expression changes based on chance alone. In the TIa vs TIb group, there are more changes than would be expected by chance. 


\section{Chromosome 3}

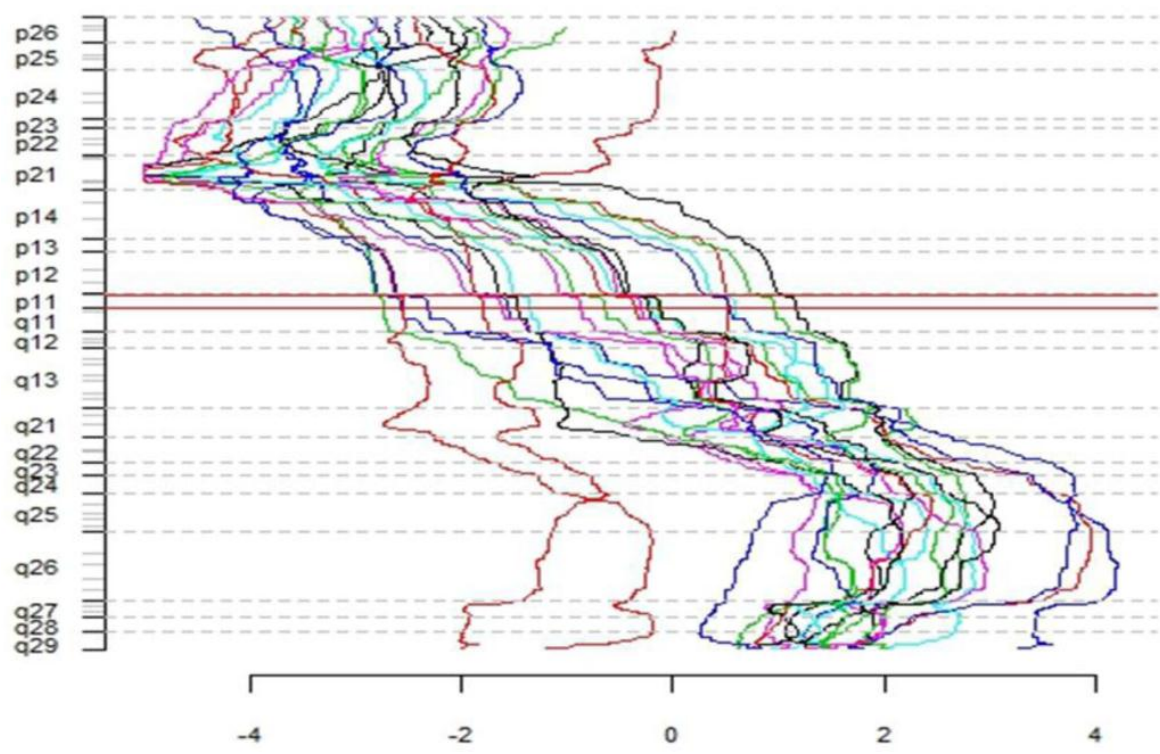

Figure 4. Comparative genome microarray analysis of chromosome 3. Note that each tumor sample has reduced expression of the short arm of chromosome 3, the site of the VHL gene

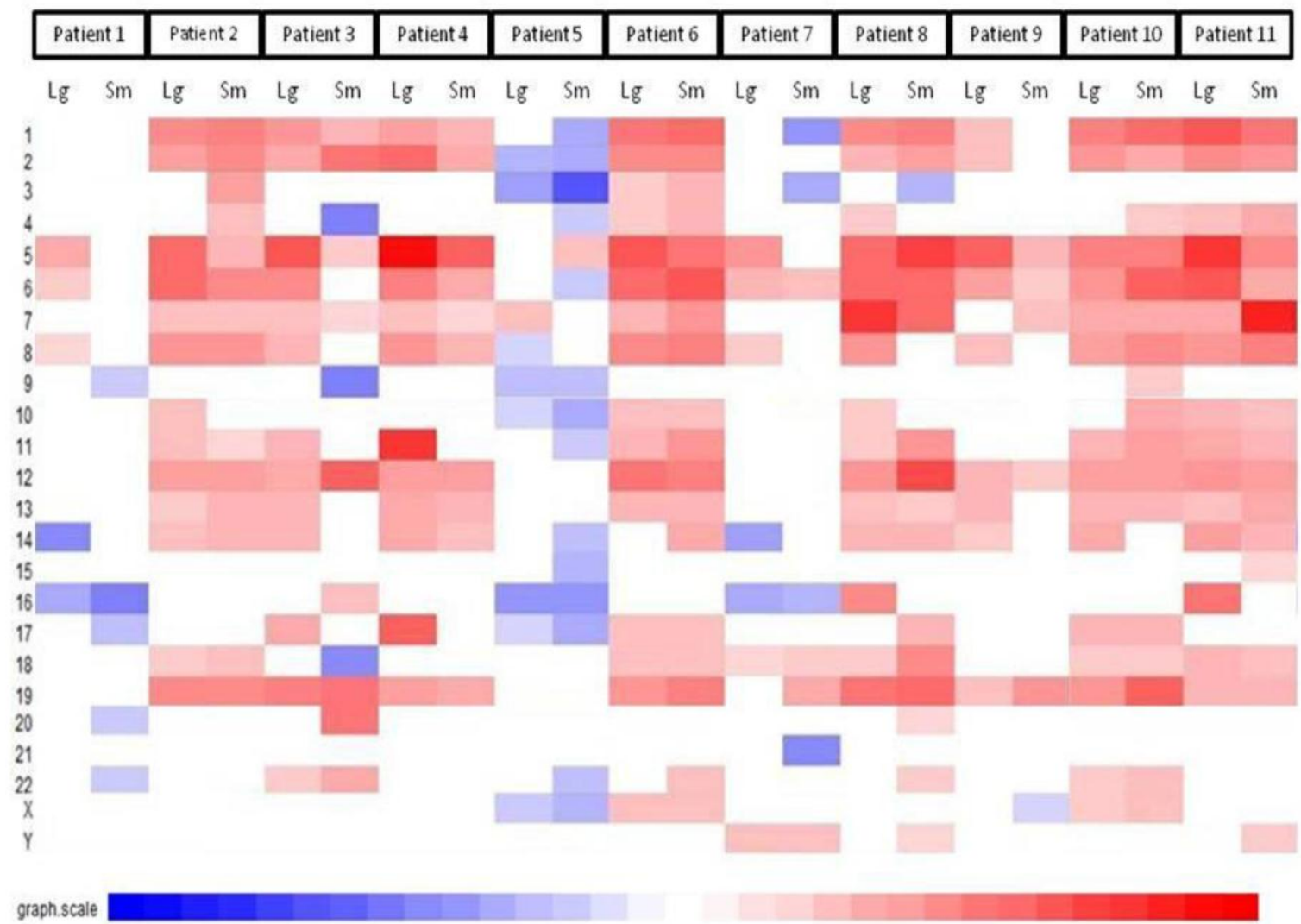

Figure 5. Comparative genomic microarray analysis - global chromosomal heat map. No consistent differences in expression on chromosomal level between large and small tumors were observed. Note that the changes between individuals are greater than changes between tumors of different size in a given individual. 


\section{Discussion}

Renal tumors in patients with von Hippel Lindau syndrome result from biallelic inactivation of the VHL gene located on chromosome 3p25 with one allele inactivated via a germline mutation and the second due to a random somatic event (7). The protein product of the VHL gene (pVHL) is a key component of an ubiquitin ligase complex that marks the alpha subunit of hypoxia inducible factor (HIF) for degradation (8). The VHL protein serves a critical role in prevention of dysregulated HIF-alpha levels (9). Downregulation of HIF-a by $\mathrm{pVHL}$ has been shown to be both necessary and sufficient for renal tumor suppression. In the absence of $\mathrm{pVHL}$ patients are prone to form tumors of the retina, CNS, kidneys, pancreas, and epididymis (10).

Among the most severe manifestations of the VHL syndrome are the renal manifestations. These patients are at risk for forming multiple bilateral clear cell renal cell carcinomas - as many as 70 clinically lesions in one kidney have been documented to be removed (11). Despite multiple foci of disease that cumulatively represent substantial tumor burden, the renal tumors in patients with VHL do not attain the ability to metastasize until the largest tumor reaches $3 \mathrm{~cm}$ (3). Similar to VHL tumors, size of sporadic renal masses is also one of the most important prognostic indicators in sporadic RCC. Frank et al estimated 5 year survival rates to be $97 \%$ for patients with $\mathrm{Tla}$ $(<4 \mathrm{~cm})$ tumors, $87 \%$ for patients with $\mathrm{T} 1 \mathrm{~b}$ tumors $(4-7$ $\mathrm{cm})$, and $71 \%$ for patients with T2 disease $(>7 \mathrm{~cm})(2)$. While the relationship between size and metastatic potential is well-established, molecular changes that are associated with tumor growth are poorly characterized.

In the present study we investigated the changes in gene expression profiles that occur with tumor growth by performing microarray analysis on small and large tumors from the same patient. Interestingly, unsupervised clustering revealed that the tumor pairs with larger volume differences clustered separately from tumor pairs with smaller volume differences. This suggests that the global transcriptome of larger tumors are more biologically dissimilar from smaller tumors within the same patient. Such observation is of potential clinical relevance as these tumors were not only resected from the same patient and the same kidney, they had the same germline mutation, and were exposed to the same environmental and host factors throughout their development. Therefore, the differences observed may be representative of biologically significant processes because the only difference between the tumors was their size.
We were also able to investigate the nature of the genetic changes on a chromosomal level between large and small tumors. CGMA was used to examine gene expression changes between tumor samples and a pool of normal renal parenchyma samples. Analysis of the gene expression from each chromosome revealed only one area that had decreased expression across all samples: $3 p$ - the location of the VHL gene. These findings are also useful as confirmatory results of our analysis, demonstrating global loss of gene expression on the $3 p$, consistent with the VHL samples studied.

In addition to demonstration of $3 p$ loss as a confirmation of our analyses, the study of cytogenetics may be particularly relevant in patients with VHL. Indeed, inactivation of $\mathrm{pVHL}$ was found to lead to defects in spindle orientation thus provoking chromosomal instability (12). Consistent with this theory, multiple chromosomal abnormalities have been reported in the literature for patients with both hereditary and sporadic ccRCC $(13,14)$. For example, Phillips et al performed cytogenetic studies on cell lines derived from vHL patients with multifocal RCC and found that higher stage tumors were associated with a greater degree of aneupolidy (14).

Notably, not all studies demonstrated genomic instability in RCC. A study of the sporadic ccRCC population failed to show an association between the degree of genomic instability and the clinical stage of renal tumors (13). Interestingly, another large scale study of 432 clear cell renal tumors supported the loss of chromosome $3 p$ as a common event in the development of ccrCC $(60 \%)$, but the prevalence of other chromosomal aberrations were much lower (14\% for loss of chromosome $4 p$ to $33 \%$ for gain of chromosome $5 q$ ). In that study, loss of chromosome 9 was the only independent predictor associated with survival (15). The lack of chromosomal changes between larger and smaller tumors in our study is inconsistent with the concept that chromosomal instability occurs in larger tumors. However, it should be noted that all our samples are derived from T1 tumors, which are associated with a very good prognosis. In contrast, the tumors studied by Phillips et al included a wider spectrum of disease, including tumors that had achieved the ability to metastasize (14). Therefore, chromosomal instability may be a late, rather than an early event, in tumor progression.

While consistent cytogenetic changes between large and small tumors were not observed, we did note significant changes in the expression of individual genes. For this analysis, our sample was split into two groups: groups where the larger tumor was less than $4 \mathrm{~cm}$ (T1a) and groups where the larger tumor 
was greater than $4 \mathrm{~cm}$ (T1b). As Figure 3 demonstrates, the number of statistically significant genetic changes between large and small tumors was no greater than would be expected by random chance when both tumors were T1a. This finding further supports the strategy used at the urologic oncology branch at the $\mathrm{NCI}$, when small tumors are surveilled until the largest tumor reaches $3 \mathrm{~cm}$ (Duffey at al). However, once the largest tumor reached T1b size (greater than $4 \mathrm{~cm}$ ), the number of statistically significant genetic changes between larger and smaller tumors becomes much greater than would be expected by random chance. This suggests that even in the absence of large cytogenetic changes, there are significant changes in gene expression as tumors grow larger. When Gene Ontology analysis was performed, it demonstrated that there was an over-representation of transcripts involved in DNA replication and in cytokine signaling, particularly IL-6. This may be clinically elevant, especially since cytokine therapy is one of the durable treatments used for advanced ccRCC.

This study represents one of the first attempts to perform microarrays on tumors derived from the same patient. Gieseg studied the gene expression profiles of smaller $(200 \mathrm{mg})$ and larger tumors (1000 $\mathrm{mm}$ ) in subcutaneously grown tumors from cell lines, and found no difference in gene expression between large and small tumors (16). This is not unexpected as a cell line represents a clonal population of cells that has been selected for high proliferative ability. Perou et al examined breast cancer samples derived from primary tumor and lymph nodes in the same patient, and found that gene expression patterns in two tumor samples from the same individual were almost always more similar to each other than either was to any other sample (17). Similarly, Chen et al examined multiple tumors from patients with HCC (18). The investigators noted that the most important factor in clustering was the individual of origin, not the size of the tumor. They found genetic differences between clonally related tumors. Consistent with our findings, the differences were particularly evident in one patient with a large tumor $(8 \mathrm{~cm})$, who had different chromosomal abnormalities than smaller tumors in the same patient $(1 \mathrm{~cm}$ and $2 \mathrm{~cm})(18)$.

The results of prior studies on tumors from the same patient corroborate our data. It appears that each individual has a very distinct pattern of transcribed genes when surveyed by gene expression microarray. Tumors have genetic aberrations, but continue to have a transcriptome that is more similar to its parental pattern than to another individual's transcriptome. Our data provide evidence of differences in gene expression between larger and small renal cell carcinoma tumors, although the number of differences may small in number in T1 renal tumors. It may not be until renal tumors are even larger that chromosome instability would be detectable.

Of note, our size criteria were chosen to reflect a clinically useful landmark. Tumors less than $4 \mathrm{~cm}$ are also known as small renal masses. As the use of CT scans increases, the incidence of these small renal masses is rising, creating a new debate about the utilization of active surveillance in select patients (19). We found that there may be a biologic basis for this practice by demonstrating few genetic differences in T1a tumors. Consistent with our findings, recent work from MSKCC studying metastatic events of sporadic RCC, found the metastatic rate negligible in patients with renal masses less than $3 \mathrm{~cm}$ (20). However, as the size of the tumors increases beyond $4 \mathrm{~cm}$, there are ongoing changes in the transcriptome, possibly reflecting the potential change in biologic aggressiveness.

The present study has a few limitations. First, the samples analyzed were archived, and there is a possibility that some of the observed changes may be due to a length of storage and procurement conditions. Second, although all patients had VHL, the different germline mutations or the second hits of the normal allele may have also be a variable, determining the expression profile of the tumors studied. Third, the sample size is small (although it includes patients treated during a period of 5 years at the NCI). Finally, we recognize that the transcriptome analysis do not always translate into proteome and biologic significance. Despite these limitations, this is the first study in RCC that compares the expression levels of large and small tumors obtained from the same individual in the same setting. Additionally, his study may provide new biologic rationale for active surveillance of small renal masses, practiced for years at the National Cancer Institute.

\section{Conclusion}

Through analysis of genetic expression profiles between larger and smaller tumors in the same patient, we found that larger tumor volume differences progressively increased global transcriptome changes. Although few changes in gene expression were appreciated on a chromosomal level, on the level on the individual transcripts, there were an increasing number of changes between large and small tumors, especially when the larger tumor was greater than $4 \mathrm{~cm}(\mathrm{~T} 1 \mathrm{~b})$. This may provide a genetic rational behind active surveillance of small renal masses in select patients and further support practice of active surveillance at the Urologic Oncology Branch at the NCI. 


\section{Acknowledgements}

We would like to thank Dr. Chandramouli Gadisetti for his help with statistical analysis.

Analyses were performed using BRB-Array Tools developed by Dr. Richard Simon and the BRB-Array Tools Development Team.

This research was supported by the Intramural Research Program of the NIH, National Cancer Institute, Center for Cancer Research

\section{Conflicts of Interest}

The authors have no conflicts of interest to report

\section{References}

1. Nickerson ML, Jaeger E, Shi $Y$, et al. Improved identification of von Hippel-Lindau gene alterations in clear cell renal tumors. Clin Cancer Res 2008; 14:4726-34.

2. Frank I, Blute ML, Leibovich BC, Cheville JC, Lohse CM, Zincke $\mathrm{H}$. Independent validation of the 2002 American Joint Committee on cancer primary tumor classification for renal cell carcinoma using a large, single institution cohort. J Urol 2005; 173:1889-92.

3. Walther MM, Choyke PL, Glenn G, et al. Renal cancer in families with hereditary renal cancer: prospective analysis of a tumor size threshold for renal parenchymal sparing surgery. J Urol 1999; 161:1475-9.

4. Simon R, Lam A, Li MC, Ngan M, Menenzes S, Zhao Y. Analysis of Gene Expression Data Using BRB-Array Tools. Cancer Inform 2007; 3:11-7.

5. Irizarry RA, $\mathrm{Wu} \mathrm{Z}$, Jaffee HA. Comparison of Affymetrix GeneChip expression measures. Bioinformatics 2006; 22:789-94.

6. Crawley JJ, Furge KA. Identification of frequent cytogenetic aberrations in hepatocellular carcinoma using gene-expression microarray data. Genome Biol 2002; 3: 0075

7. Linehan WM, Bratslavsky G, Pinto PA, et al. Molecular diagnosis and therapy of kidney cancer. Annu Rev Med; 2010; 61:329-43.

8. Linehan WM, Walther MM, Zbar B. The genetic basis of cancer of the kidney. J Urol 2003; 170:2163-72.

9. Lubensky IA, Gnarra JR, Bertheau P, Walther MM, Linehan WM, Zhuang Z. Allelic deletions of the VHL gene detected in multiple microscopic clear cell renal lesions in von Hippel-Lindau disease patients. Am J Pathol 1996; 149:2089-94.

10. Kaelin WGJr. The von Hippel-Lindau tumor suppressor protein and clear cell renal carcinoma. Clin Cancer Res 2007; 13:680s-4s.

11. Fadahunsi AT, Sanford T, Linehan WM, Pinto PA, Bratslavsky G. Feasibility and outcomes of partial nephrectomy for resection of at least 20 tumors in a single renal unit. J Urol 2011; 185:49-53.

12. Thoma CR, Toso A, Gutbrodt KL, et al. VHL loss causes spindle misorientation and chromosome instability. Nat Cell Biol 2009; 11:994-1001.

13. Thrash-Bingham CA, Salazar H, Freed JJ, Greenberg RE, Tartof KD. Genomic alterations and instabilities in renal cell carcinomas and their relationship to tumor pathology. Cancer Res 1995; 55:6189-95.

14. Phillips JL, Ghadimi BM, Wangsa D, et al. Molecular cytogenetic characterization of early and late renal cell carcinomas in von Hippel-Lindau disease. Genes Chromosomes Cancer 2001; 31:1-9.
15. Klatte $\mathrm{T}$, Rao PN, de Martino M, et al. Cytogenetic profile predicts prognosis of patients with clear cell renal cell carcinoma. J Clin Oncol 2009; 27:746-53.

16. Gieseg MA, Man MZ, Gorski NA, Madore SJ, Kaldjian EP, Leopold WR. The influence of tumor size and environment on gene expression in commonly used human tumor lines. BMC Cancer 2004; 4:35.

17. Perou CM, Sorlie T, Eisen MB, et al. Molecular portraits of human breast tumours. Nature 2000; 406:747-52.

18. Chen X, Cheung ST, So S, et al. Gene expression patterns in human liver cancers. Mol Biol Cell 2002; 13:1929-39.

19. Jewett MA, Zuniga A. Renal tumor natural history: the rationale and role for active surveillance. Urol Clin North Am 2008; 35:627-34.

20. Thompson RH, Hill JR, Babayev Y, et al. Metastatic renal cell carcinoma risk according to tumor size. J Urol 2009; 182:41-5. 